\title{
Sub-Femto-g Free Fall for Space-Based Gravitational Wave Observatories: LISA Pathfinder Results
}

M. Armano, ${ }^{1}$ H. Audley, ${ }^{2}$ G. Auger, ${ }^{3}$ J. T. Baird, ${ }^{4}$ M. Bassan,${ }^{5}$ P. Binetruy, ${ }^{3}$ M. Born, ${ }^{2}$ D. Bortoluzzi,${ }^{6}$ N. Brandt, ${ }^{7}$ M. Caleno, ${ }^{8}$ L. Carbone, ${ }^{9}$ A. Cavalleri, ${ }^{10}$ A. Cesarini, ${ }^{9}$ G. Ciani, ${ }^{9,}$ G. Congedo, ${ }^{9, \$}$ A. M. Cruise, ${ }^{11}$ K. Danzmann, ${ }^{2}$ M. de Deus Silva, ${ }^{1}$ R. De Rosa, ${ }^{12}$ M. Diaz-Aguiló, ${ }^{13}$ L. Di Fiore, ${ }^{14}$ I. Diepholz, ${ }^{2}$ G. Dixon, ${ }^{11}$ R. Dolesi, ${ }^{9}$ N. Dunbar, ${ }^{15}$ L. Ferraioli, ${ }^{16}$ V. Ferroni, ${ }^{9}$ W. Fichter ${ }^{17}$ E. D. Fitzsimons, ${ }^{18}$ R. Flatscher, ${ }^{7}$ M. Freschi, ${ }^{1}$ A. F. García Marín, ${ }^{2,8}$ C. García Marirrodriga, ${ }^{8}$ R. Gerndt, ${ }^{7}$ L. Gesa, ${ }^{13}$ F. Gibert, ${ }^{9}$ D. Giardini, ${ }^{16}$ R. Giusteri, ${ }^{9}$ F. Guzmán, ${ }^{2, \|}$ A. Grado, ${ }^{19}$ C. Grimani, ${ }^{20}$ A. Grynagier, ${ }^{17,}$ J. Grzymisch, ${ }^{8}$ I. Harrison, ${ }^{21}$ G. Heinzel, ${ }^{2}$ M. Hewitson, ${ }^{2}$ D. Hollington, ${ }^{4}$ D. Hoyland, ${ }^{11}$ M. Hueller, ${ }^{9}$ H. Inchauspé, ${ }^{3}$ O. Jennrich, ${ }^{8}$ P. Jetzer, ${ }^{22}$ U. Johann, ${ }^{7}$ B. Johlander, ${ }^{8}$ N. Karnesis, ${ }^{2}$ B. Kaune, ${ }^{2}$ N. Korsakova, ${ }^{2}$ C. J. Killow, ${ }^{23}$ J. A. Lobo, ${ }^{13, *}$ I. Lloro, ${ }^{13}$ L. Liu, ${ }^{9}$ J. P. López-Zaragoza, ${ }^{13}$ R. Maarschalkerweerd,${ }^{21}$ D. Mance, ${ }^{16}$ V. Martín, ${ }^{13}$ L. Martin-Polo, ${ }^{1}$ J. Martino, ${ }^{3}$ F. Martin-Porqueras, ${ }^{1}$ S. Madden,${ }^{8}$ I. Mateos,${ }^{13}$ P. W. McNamara, ${ }^{8}$ J. Mendes,${ }^{21}$ L. Mendes, ${ }^{1}$ A. Monsky, ${ }^{2,8}$ D. Nicolodi, ${ }^{9,}$ M. Nofrarias, ${ }^{13}$ S. Paczkowski, ${ }^{2}$ M. Perreur-Lloyd, ${ }^{23}$ A. Petiteau, ${ }^{3}$ P. Pivato, ${ }^{9}$ E. Plagnol, ${ }^{3}$ P. Prat, ${ }^{3}$ U. Ragnit, ${ }^{8}$ B. Raïs, ${ }^{3}$ J. Ramos-Castro, ${ }^{24}$ J. Reiche, ${ }^{2}$ D. I. Robertson, ${ }^{23}$ H. Rozemeijer, ${ }^{8}$ F. Rivas,${ }^{13}$ G. Russano, ${ }^{9}$ J. Sanjuán, ${ }^{13, \dagger}$ P. Sarra, ${ }^{25}$ A. Schleicher, ${ }^{7}$ D. Shaul, ${ }^{4}$ J. Slutsky, ${ }^{26}$ C. F. Sopuerta,${ }^{13}$ R. Stanga, ${ }^{27}$ F. Steier,,${ }^{2,}$ T. Sumner ${ }^{4}$ D. Texier, ${ }^{1}$ J. I. Thorpe ${ }^{26}{ }^{26}$ C. Trenkel,${ }^{15}$ M. Tröbs, ${ }^{2}$ H. B. Tu, ${ }^{9,}{ }^{,+}$D. Vetrugno, ${ }^{9}$ S. Vitale, ${ }^{9}$ V. Wand,${ }^{2, \$ \S}$ G. Wanner, ${ }^{2}$ H. Ward, ${ }^{23}$ C. Warren, ${ }^{15}$ P. J. Wass ${ }^{4}$ D. Wealthy, ${ }^{15}$ W. J. Weber, ${ }^{9}$ L. Wissel, ${ }^{2}$ A. Wittchen, ${ }^{2}$ A. Zambotti, ${ }^{6}$ C. Zanoni, ${ }^{6}$ T. Ziegler, ${ }^{7}$ and P. Zweifel ${ }^{16}$

${ }^{1}$ European Space Astronomy Centre, European Space Agency, Villanueva de la Cañada, 28692 Madrid, Spain

${ }^{2}$ Albert-Einstein-Institut, Max-Planck-Institut für Gravitationsphysik und Leibniz Universität Hannover, Callinstraße 38, 30167 Hannover, Germany

${ }^{3} A P C$, Université Paris Diderot, CNRS/IN2P3, CEA/lrfu, Obs de Paris, Sorbonne Paris Cité, France

${ }^{4}$ High Energy Physics Group, Physics Department, Imperial College London, Blackett Laboratory, Prince Consort Road, London, SW7 2BW, United Kingdom

${ }^{5}$ Dipartimento di Fisica, Università di Roma "Tor Vergata", and INFN, sezione Roma Tor Vergata, I-00133 Roma, Italy

${ }^{6}$ Department of Industrial Engineering, University of Trento, via Sommarive 9, 38123 Trento, and Trento Institute for Fundamental Physics and Applications/INFN, Italy

${ }^{7}$ Airbus Defence and Space, Claude-Dornier-Strasse, 88090 Immenstaad, Germany

${ }^{8}$ European Space Technology Centre, European Space Agency, Keplerlaan 1, 2200 AG Noordwijk, Netherlands

${ }^{9}$ Dipartimento di Fisica, Università di Trento and Trento Institute for Fundamental Physics and Applications/INFN, 38123 Povo, Trento, Italy

${ }^{10}$ Istituto di Fotonica e Nanotecnologie, CNR-Fondazione Bruno Kessler, I-38123 Povo, Trento, Italy

${ }^{11}$ The School of Physics and Astronomy, University of Birmingham, Birmingham, United Kingdom

${ }^{12}$ Dipartimento di Fisica, Università di Napoli "Federico II" and INFN-Sezione di Napoli, I-80126, Napoli, Italy

${ }^{13}$ Institut de Ciències de l'Espai (CSIC-IEEC), Campus UAB, Carrer de Can Magrans s/n, 08193 Cerdanyola del Vallès, Spain

${ }^{14}$ INFN-Sezione di Napoli, I-80126, Napoli, Italy

${ }^{15}$ Airbus Defence and Space, Gunnels Wood Road, Stevenage, Hertfordshire, SG1 2AS, United Kingdom

${ }^{16}$ Institut für Geophysik, ETH Zürich, Sonneggstrasse 5, CH-8092, Zürich, Switzerland

${ }^{17}$ Universitt Stuttgart, Institut fr Flugmechanik und Flugregelung, Pfaffenwaldring 27, 70569 Stuttgart, Germany

${ }^{18}$ The UK Astronomy Technology Centre, Royal Observatory, Edinburgh, Blackford Hill, Edinburgh, EH9 3HJ, United Kingdom

${ }^{19}$ INAF Osservatorio Astronomico di Capodimonte, I-80131 Napoli, Italy and INFN sezione di Napoli, I-80126 Napoli, Italy

${ }^{20}$ DISPEA, Università di Urbino "Carlo Bo", Via S. Chiara, 2761029 Urbino/INFN, Italy

${ }^{21}$ European Space Operations Centre, European Space Agency, 64293 Darmstadt, Germany

${ }^{22}$ Physik Institut, Universität Zürich, Winterthurerstrasse 190, CH-8057 Zürich, Switzerland

${ }^{23}$ SUPA, Institute for Gravitational Research, School of Physics and Astronomy, University of Glasgow, Glasgow, G12 8QQ, United Kingdom

${ }^{24}$ Departament d'Enginyeria Electrònica, Universitat Politècnica de Catalunya, 08034 Barcelona, Spain

${ }^{25}$ CGS S.p.A, Compagnia Generale per lo Spazio, Via Gallarate, 150-20151 Milano, Italy

${ }^{26}$ Gravitational Astrophysics Lab, NASA Goddard Space Flight Center, 8800 Greenbelt Road, Greenbelt, Maryland 20771, USA

${ }^{27}$ Dipartimento di Fisica ed Astronomia, Università degli Studi di Firenze and INFN-Sezione di Firenze, I-50019 Firenze, Italy (Received 4 May 2016; published 7 June 2016)

Published by the American Physical Society under the terms of the Creative Commons Attribution 3.0 License. Further distribution of this work must maintain attribution to the author(s) and the published article's title, journal citation, and DOI. 
We report the first results of the LISA Pathfinder in-flight experiment. The results demonstrate that two free-falling reference test masses, such as those needed for a space-based gravitational wave observatory like LISA, can be put in free fall with a relative acceleration noise with a square root of the power spectral density of $5.2 \pm 0.1 \mathrm{fm} \mathrm{s}^{-2} / \sqrt{\mathrm{Hz}}$, or $(0.54 \pm 0.01) \times 10^{-15} \mathrm{~g} / \sqrt{\mathrm{Hz}}$, with $g$ the standard gravity, for frequencies between 0.7 and $20 \mathrm{mHz}$. This value is lower than the LISA Pathfinder requirement by more than a factor 5 and within a factor 1.25 of the requirement for the LISA mission, and is compatible with Brownian noise from viscous damping due to the residual gas surrounding the test masses. Above $60 \mathrm{mHz}$ the acceleration noise is dominated by interferometer displacement readout noise at a level of $(34.8 \pm 0.3) \mathrm{fm} / \sqrt{\mathrm{Hz}}$, about 2 orders of magnitude better than requirements. At $f \leq 0.5 \mathrm{mHz}$ we observe a low-frequency tail that stays below $12 \mathrm{fm} \mathrm{s}^{-2} / \sqrt{\mathrm{Hz}}$ down to $0.1 \mathrm{mHz}$. This performance would allow for a space-based gravitational wave observatory with a sensitivity close to what was originally foreseen for LISA.

DOI: 10.1103/PhysRevLett.116.231101

\section{INTRODUCTION}

LISA Pathfinder [1] (LPF) is a European Space Agency mission launched on December 3, 2015 and dedicated to an end-to-end experimental demonstration of the free fall of test masses (TMs) at the level required for a future space-based gravitational wave $(\mathrm{GW})$ observatory, such as LISA [2]. The TMs in LISA are the reference bodies at the ends of each interferometer arm, and need to be free from spurious acceleration, $g$, relative to their local inertial frame, any such acceleration being in direct competition with the tidal deformations caused by GWs. The LPF spacecraft has two LISA TMs at the ends of a short interferometer arm, insensitive to GWs because of the reduced length, but sensitive to the differential acceleration, $\Delta g$, of the TMs arising from parasitic forces.

The logic of the mission is to perform these experiments with hardware that can be transferred to the final observatory and with only a slight relaxation of the test requirements imposed by programmatic constraints. While the LISA design specifies a single TM acceleration noise with the square root of the power spectral density, a quantity we call amplitude spectral density (ASD), below $3 \mathrm{fm} \mathrm{s}^{-2} / \sqrt{\mathrm{Hz}}$ at $0.1 \mathrm{mHz}$, the lowest frequency of its measurement band, the LPF differential acceleration noise requirement was relaxed to $30 \mathrm{fm} \mathrm{s}^{-2} / \sqrt{\mathrm{Hz}}$ at $1 \mathrm{mHz}$. Increasing the minimum frequency to $1 \mathrm{mHz}$ reduced the time and difficulty of industrial testing, and the increase in the allowed noise floor-a relaxation of $\sim 7$ from LISA - allows for limitations inherent to a single spacecraft experiment, as discussed below. LPF is also required to demonstrate a laser interferometric readout of the relative TM motion, with noise below $9 \mathrm{pm} / \sqrt{\mathrm{Hz}}$ above $3 \mathrm{mHz}$, approaching the performance required for the local test mass position readout in LISA.

Following launch and a 50 day cruise phase to its final orbit around the L1 Lagrange point of the Sun-Earth system, LISA Pathfinder separated from its propulsion module. Commissioning of the instrument followed, with the release of the two TMs into free fall 74 days after launch in mid-February. The mission began science operations on March 1, 2016, 89 days after launch, and has been operating smoothly ever since.

The best example of the results obtained to date is the noise measurement shown in Fig. 1, with the ASD of the differential TM acceleration $\Delta g$ compared to the LPF and LISA requirements. The ASD of the noise, $S_{\Delta g}^{1 / 2}$, is below LPF requirements by more than a factor 5 at the $1 \mathrm{mHz}$ lower frequency limit. In addition $S_{\Delta g}^{1 / 2}$ is below the LISA requirement at frequencies higher than $10 \mathrm{mHz}$ and is above the requirement by no more than a factor 1.4 at all frequencies between 0.5 and $10 \mathrm{mHz}$. This factor has further reduced to 1.25 , measured in a later, shorter noise run on day 146 (see below). Below $0.5 \mathrm{mHz}$, the noise increases slightly ending up above the LISA requirement by roughly a factor 3 at $0.1 \mathrm{mHz}$. The acceleration noise ASD is clearly dominated by two main effects which cross over around $60 \mathrm{mHz}$; a discussion of the two effects is given later in the text. This Letter reports on the first 55 days of science operations, including a description of the differential acceleration measurements performed, the main results obtained so far, and their implications for a largescale space-based GW observatory. In-depth reports on the wealth of dedicated experiments of LPF, many of which are still being performed, will be reported in separate papers.

\section{DESCRIPTION OF THE TEST}

Interferometric gravitational-wave detectors work on the principle that, in curved spacetime, two distant free-falling observers exchanging an electromagnetic beam observe a time-varying difference between their respective measurements of the beam frequency [3]. This effect is indistinguishable from that caused by the Doppler effect if the observers accelerate relative to their respective inertial frames, meaning that spurious random forces can limit the sensitivity of interferometric gravitational-wave detectors.

In LISA, the most studied concept for a space-based GW detector [4], the two free-falling observers are replaced by a pair of TMs, each in free fall inside a separate spacecraft, 


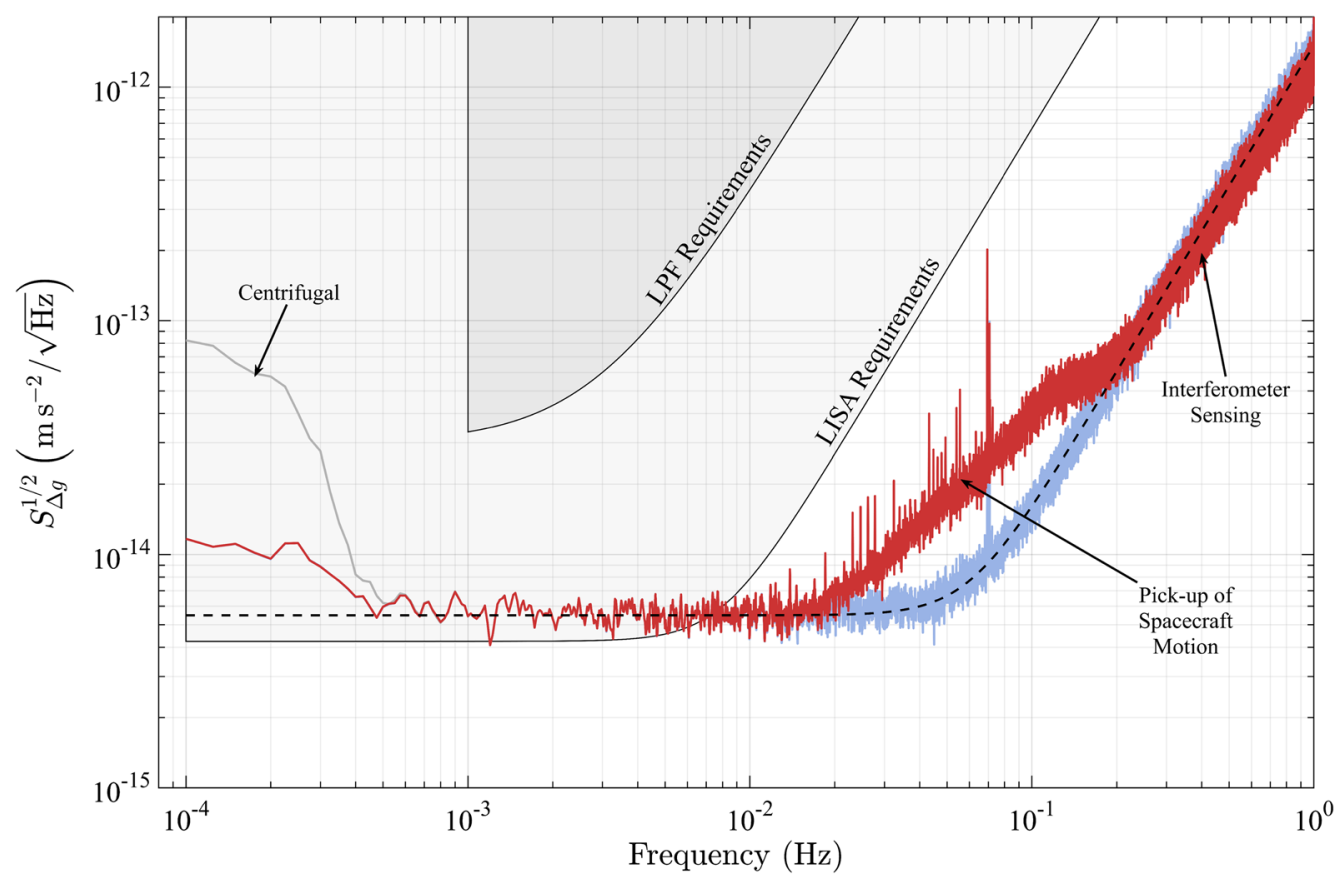

FIG. 1. Gray: ASD of $\Delta g, S_{\Delta g}^{1 / 2}(f)$, measured for 6.5 days starting 127 days after launch. The ASD is the result of averaging 26 periodograms of $40000 \mathrm{~s}$ each, which results in a relative error $(1 \sigma)$ of $10 \%$ in $S_{\Delta g}^{1 / 2}$. The effective spectral resolution, set by the spectral window, is $\Delta f \simeq \pm 50 \mu \mathrm{Hz}$. The absolute calibration of the measurement is better than $5 \%$. Red: ASD of the same time series after correction for the centrifugal force (visible at the lowest frequencies). Light blue: ASD after correction for the pickup of spacecraft motion by the interferometer (IFO), visible in the $20-200 \mathrm{mHz}$ range. Dashed smooth black line: $S_{\Delta g}(f)=S_{0}+S_{\mathrm{IFO}}(2 \pi f)^{4}$ with $S_{0}^{1 / 2}=(5.57 \pm 0.04) \mathrm{fm} \mathrm{s}^{-2} / \sqrt{\mathrm{Hz}}$ and $S_{\mathrm{IFO}}^{1 / 2}=(34.8 \pm 0.3) \mathrm{fm} / \sqrt{\mathrm{Hz}}$. Note that the level of $S_{0}$ has decreased further in subsequent measurements, as quoted in the abstract and shown in Fig. 3. Shaded areas: LISA and LISA Pathfinder requirements for $\Delta g$. The LISA single test-mass acceleration requirement [2] has been multiplied by $\sqrt{2}$ to be presented here as a differential acceleration.

exchanging a laser beam over a few million kilometres. To achieve the full science objectives of LISA, the ASD of spurious random accelerations of the TMs must be limited to $S_{g}^{1 / 2}(f) \leq 3 \mathrm{fm} \mathrm{s}^{-2} / \sqrt{\mathrm{Hz}} \times \sqrt{1+(f / 8 \mathrm{mHz})^{4}}$ within the frequency band of the detector, $0.1 \mathrm{mHz} \leq f \leq 1 \mathrm{~Hz}$. The $f^{2}$ relaxation for $f \geq 8 \mathrm{mHz}$ arises because at those frequencies the noise is expected to be dominated by white interferometer displacement noise that, when converted to equivalent acceleration, scales like $f^{2}$. The requirement should be given in terms of the differential acceleration, $\Delta g$, between the two test masses. However, as the two spacecraft are separated by a large distance, force fluctuations around each TM are assumed to be incoherent and $S_{\Delta g}^{1 / 2}=\sqrt{2} S_{g}^{1 / 2}$.

At frequencies below $1 \mathrm{~Hz}$, there is currently no realistic possibility to reach such a level of free fall in a ground based laboratory. The main problems are the large acceleration of the laboratory relative to a local inertial frame and low-frequency terrestrial gravitational noise. This pushes low-frequency GW detectors to space but also prevents an end-to-end experimental demonstration of the required free-fall performance in a terrestrial laboratory, leading to the need for the LISA Pathfinder mission, whose requirements for the ASD of $\Delta g$ have been set at $S_{\Delta g}^{1 / 2}(f) \leq$
$30 \mathrm{fm} \mathrm{s}^{-2} / \sqrt{\mathrm{Hz}} \times \sqrt{1+(f / 3 \mathrm{mHz})^{4}}$ within the frequency band $1 \mathrm{mHz} \leq f \leq 30 \mathrm{~Hz}$. Note that for LPF the cross-over frequency to the $f^{2}$ branch $(3 \mathrm{mHz})$, corresponds to the value used in the earliest LISA concept [4], while the change to $8 \mathrm{mHz}$ results from the latest studies [2]. This difference has no practical impact on the work presented here.

\section{A. The instrument}

The core instrument of LPF [5], consists of two quasicubic test masses, of size $(46.000 \pm 0.005) \mathrm{mm}$ and mass $M=(1.928 \pm 0.001) \mathrm{kg}$, formed from a high-purity goldplatinum alloy. During science operations, these masses are in free fall inside a single spacecraft with their centers separated by a nominal distance of $(376.00 \pm 0.05) \mathrm{mm}$ along a line that we take as the $x$ axis (see Fig. 2 and Ref. [6]). Each TM is contained within an electrode housing [7], which serves as an electrostatic shield in addition to a 6 degree-of-freedom sensor and electrostatic force actuator, with gaps around the mechanically and electrically isolated TM of 2.9-4 mm on the different axes. Charge accumulated by the TMs due to cosmic rays is removed by a UV light discharge system [8].

DC and slowly varying electrostatic forces are applied with dedicated audio frequency voltages between 60 and 


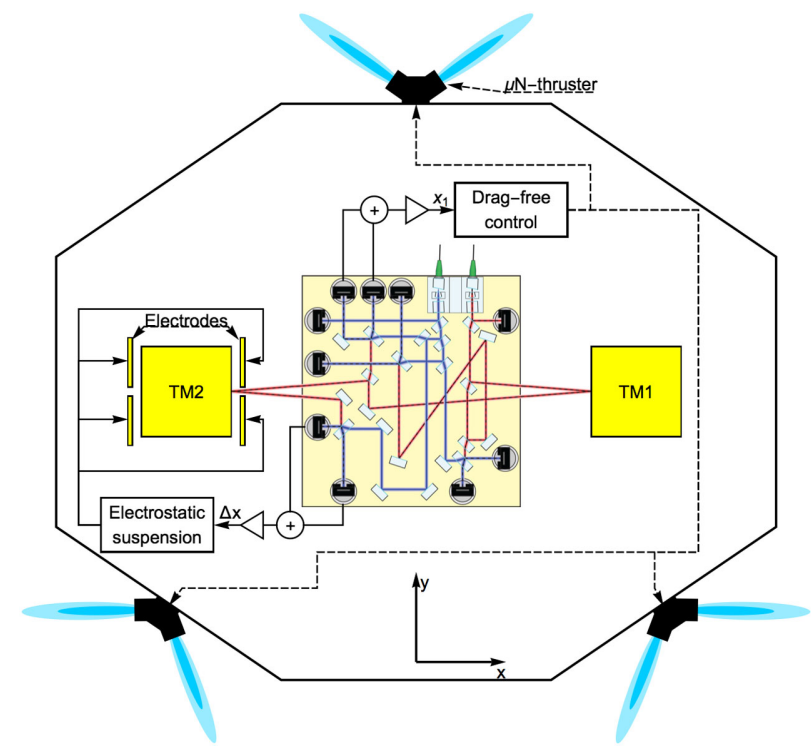

FIG. 2. A schematic of LPF. The figure shows TM1, TM2, and the optical bench beam paths for measuring $\Delta x$ and $x_{1}$. The measurement of $\Delta x$ drives the electrostatic suspension of TM2, which applies the necessary electrostatic forces by means of the electrodes represented by the four gold plates facing TM2. All other electrodes surrounding the TMs are not shown. The measurement of $x_{1}$ drives the drag-free control loop that uses the micronewton thrusters to exert forces on the spacecraft. The figure depicts the $x$ and $y$ axes we use in this Letter, while $z$ is normal to the figure.

$270 \mathrm{~Hz}$, depending on the degree of freedom, which avoids the mixing of low-frequency electrical noise into force noise in the measurement band [5].

Voltages are simultaneously applied to all electrodes, with commanded carrier amplitudes adjusted to produce any desired force with amplitude up to a fixed limitreferred to here as the actuation "authority"-while holding the resulting electrostatic force gradient (or stiffness) constant. Force noise associated with this electrostatic suspension depends on the applied force levels but also on the force and torque authorities [9]. To limit this noise contribution we have reduced the authority for the critical $x$ and $\phi$ (rotation around $z$, see Fig. 2) degrees of freedom during science operations.

Our science signal is in the differential displacement of the two TM along the $x$ axis, $\Delta x \equiv x_{2}-x_{1}$, and this is measured by a dedicated heterodyne laser interferometer [10-12]. A second interferometer measures the displacement of TM1 relative to the spacecraft, $x_{1}$. Both interferometers have quadrant diode wavefront sensing, allowing measurement of both TM $\phi$ and $\eta$ rotations around $z$ and $y$ (see Fig. 2), respectively.

The TMs and the spacecraft constitute a three-body dynamical system in which we control all 12 degrees of freedom of the relative motion of the TMs and spacecraft. At frequencies below $1 \mathrm{mHz}$ we also control the orientation of the spacecraft relative to the J2000 reference frame.
Describing the details of this system goes beyond the scope of this Letter [13]. Here we just recall the logic of the control scheme for the $x$ axis. Along this axis the spacecraft is forced to follow TM1 to keep it in the center of its electrode housing. For this, $x_{1}$ is fed to a controller residing within the onboard computer. The controller commands a set of coldgas micronewton thrusters to ensure the spacecraft follows TM1 (see Fig. 2). This scheme, called drag-free control [13], is also an essential element of the LISA spacecraft control, and is therefore one of the key elements under test.

A second control loop forces TM2 to stay at a fixed distance from TM1 and thus centered in its own electrode housing; we call this electrostatic suspension. The controller, which operates at low frequencies compared to the LPF measurement band, uses $\Delta x$ as an error signal, and commands the electrostatic actuation system to generate the necessary forces on TM2. This controller is required because any static difference in force between the TMs would accelerate TM2 relative to TM1, eventually pushing it into contact with its electrode housing.

\section{B. Comparing to LISA}

Electrostatic suspension along the sensitive axis is not needed in LISA as the two TMs at the ends of the same arm can be followed independently by their respective spacecraft along the direction of the laser beam [2]. This results in both TMs staying centered along the beam direction in their respective housings.

In LPF, this necessary applied control force must be subtracted from the measured acceleration to give the relevant $\Delta g$. The subtraction of the commanded force time series, $g_{c}(t)$, and its calibration, is discussed in the next section. This also introduces potentially important actuation force noise, that is not present in LISA, into our LPF data. Note that everywhere in this Letter forces are expressed per unit mass and that we treat signals as continuous, as the $10 \mathrm{~Hz}$ sampling frequency is much larger than the maximum frequency of interest of about $0.1 \mathrm{~Hz}$.

The electrostatic suspension is not the only difference with LISA. As all measurements and controls in LPF are performed relative to the spacecraft reference frame, the component of the centrifugal force from rotation around $y$ and $z$ is directly picked up as an effective differential force. This force is quite relevant for frequencies below $0.5 \mathrm{mHz}$ as the input to the attitude controller of the spacecraft is a set of autonomous star trackers with relatively high sensing noise of about $10^{-3} \mathrm{rad} / \sqrt{\mathrm{Hz}}$ at $0.1 \mathrm{mHz}$. This noise causes a significant noisy angular velocity $\Omega_{n}(t)$ relative to the local inertial frame. $\Omega_{n}(t)$ adds to the quasistatic part, $\Omega_{\mathrm{qs}}(t)$, of a few degrees per day, needed to keep the communication antenna pointed toward the Earth. As the centrifugal force is quadratic in the angular velocity, it acquires, to first order in $\Omega_{n}$, a noisy component proportional to $\Omega_{n}(t) \Omega_{\mathrm{qs}}(t)$. Nominally, this effect will not be present in LISA where the two TMs at the end of each 
arm are in free fall along the direction of the beam. Geometrical imperfections may reintroduce some coupling, but as the attitude control in LISA uses the wave fronts of the incoming laser beams as a reference, the resulting angular noise is expected to be smaller than $10 \mathrm{nrad} / \sqrt{\mathrm{Hz}}$ [14]. On LPF we thus correct the data by subtracting the centrifugal force time series, $g_{\Omega}(t)$, calculated from the measured spacecraft angular velocity, as discussed in the next section.

The third relevant difference is in the coupling of the two masses to the spacecraft motion through the static force gradients at the nominal positions of the two TMs [5]. In LISA, the TMs at the ends of one arm are coupled to their own distant spacecraft, while in LPF they share a common spacecraft. The quasielastic coupling forces can however be calibrated and subtracted, both on LPF and LISA [3], by the local interferometer measurement of the relative spacecraft motion and dedicated experiments to measure the force gradients. For LPF we define the force gradients, or stiffnesses, (per unit mass) in the nominal TM1 and TM2 positions, $-\omega_{1}^{2}$ and $-\omega_{2}^{2}$, in terms of the corresponding natural harmonic oscillator angular frequencies. In our case the force gradients, dominated by electrostatics and local gravitation, give rise to an unstable equilibrium at the nominal TM position, with $\omega_{1}^{2}$ and $\omega_{2}^{2}$ both negative.

\section{Observables, system calibration, and data processing}

We calculate the main observable of LPF, $\Delta g$, as

$$
\begin{aligned}
\Delta g(t) \equiv & \Delta \ddot{x}(t)+\omega_{2}^{2} \Delta x(t)+\Delta \omega_{12}^{2} x_{1}(t) \\
& -g_{c}(t)-g_{\Omega}(t),
\end{aligned}
$$

where $\Delta \ddot{x}(t)$ is the numerical second time derivative of $\Delta x(t)$ and where we have defined the differential stiffness, $\Delta \omega_{12}^{2} \equiv \omega_{2}^{2}-\omega_{1}^{2}$, which couples spacecraft motion into $\Delta g$. In addition to the subtracted control force, $g_{c}(t)$, stiffness terms are calculated from measured displacement time series, $\Delta x(t)$ and $x_{1}(t)$. The centrifugal correction, $g_{\Omega}$, is calculated from the angular velocity noise, obtained by integrating the torque that the controller has to apply to the two TMs to hold them with fixed orientation relative to the spacecraft. In addition, we measure the quasistatic part of the angular velocity from the star trackers. From these two measurements, and from the nominal separation between the TMs, we generate the time series of the centrifugal force.

The contribution from centrifugal force has varied by more than an order of magnitude over the course of the 55 days of operations presented here. This variation arises from the fact that both the angular velocity of the spacecraft and the noise in the angular measurements change with time.

The centrifugal correction does not introduce significant excess noise. We estimate this noise by using the difference between the integrated torques on TM2 and TM1, instead of their mean, as the difference is insensitive to spacecraft motion but still sensitive to measurement noise. We find that the excess noise for the run in Fig. 1 is smaller than $1 \mathrm{fm} \mathrm{s}^{-2} / \sqrt{\mathrm{Hz}}$. We also find that the residual lowfrequency noise in the final run on day 146, where the centrifugal correction is negligible due to orbital dynamics, is compatible with that of Fig. 1.

The data series in Eq. (1) are calibrated as follows. We inject a guidance signal into the electrostatic suspension loop consisting of a discrete set of sinusoidal modulations at frequencies between 1 and $30 \mathrm{mHz}$ with amplitudes of around $1 \mathrm{~nm}$. In addition to modulating the relative displacements of TM2 relative to TM1 and to the spacecraft, this signal also introduces relatively large commanded forces, $g_{c}(t)$. We also inject similar guidance signals into the drag-free loop to excite the motion of the spacecraft relative to TM1 and to calibrate $\Delta \omega_{12}^{2}$. To extract the calibration parameters we fit $\Delta \ddot{x}(t)$ to the following simple model:

$$
\Delta \ddot{x}(t)=(1+\lambda) g_{c}(t)-\omega_{2}^{2} \Delta x(t)-\Delta \omega_{12}^{2} x_{1}(t),
$$

leaving $\lambda, \omega_{2}^{2}$, and $\Delta \omega_{12}^{2}$ as free parameters in the fit. The quality of the fit is generally good, as the ASD of the residuals agrees with that of the data series measured in the absence of the injected signals.

We find $\lambda=0.012 \pm 0.005$, showing that, within about $2 \sigma$, the calibration of $g_{c}$ is consistent with the independent calibration of $\Delta \ddot{x}(t)$. The first of these independent calibrations is based on the absolute voltage calibration of the electronics together with a capacitive model derived from finite element calculations and tested with a torsion pendulum [15]; the second is based on the combination of the absolute calibration of the interferometer's response, also measured on ground, of the onboard clock, and finally of the mass of the TMs. This also shows that all these calibrations have not been affected significantly by launch and by on-orbit operations.

As an independent confirmation of the absolute calibration of forces we have also modulated the power of the laser beam that hits the TMs. The measured peak value of the force resulting from the photon pressure agrees with that calculated from the power modulation to an accuracy of about $20 \%$.

The measured stiffness values agree at a $10 \%$ level with the expected values from our model of spacecraft self-gravity [16] and electrostatic force gradients, the latter including both sensing and applied $x$ and $\phi$ actuation voltages. Relevant values for the run analyzed in Fig. 1, employing the minimum actuation authorities in which gravitation is the dominant contribution, are $\omega_{2}^{2}=$ $(-525 \pm 30) \times 10^{-9} / \mathrm{s}^{2}$ and $\Delta \omega_{12}^{2}$ consistent with zero.

The calibration of the centrifugal force relies on the same calibration of the actuation electronics performed for $g_{c}$, the 
absolute angular calibration of star trackers, the calibration of the distance between TMs, and finally on the moments of inertia of the TMs. The accuracy is limited by the errors in torque calibration and can be estimated to be of the order $5 \%$.

After calibration of the system, the estimate of the ASD of $\Delta g$ is made on several noise-only runs, each lasting many days, during which no calibration forces were applied to the TMs. Data from these runs were processed to form a set of $\Delta g(t)$ time series according to Eq. (1). The ASD of these data series was estimated using Welch's averaged periodogram method $[17,18]$, using $50 \%$ overlapping data stretches and a Blackmann-Harris spectral window. The first four frequency bins of the averaged periodogram, including the one at dc, are discarded, as they are heavily biased by the spectral leakage from very low-frequency noise.

\section{RESULTS AND DISCUSSION}

Eleven noise runs, with durations ranging from 1.5 days up to 2 weeks, giving a total of more than 650 hours, were performed in the first 55 days of operations, all of which ran to completion smoothly in their science measurement configuration. During these measurement periods only two significant "fast" glitches (about $100 \mathrm{~s}$ duration), and one long period (several hours duration) of nonstationarity were observed. These outliers have been excluded from the analysis reported in Fig. 3.

With the noise slowly decreasing over most of the frequency range, the ASD in Fig. 1 uses data from the last continuous stretch of data long enough to give 10\% statistical precision at frequencies down to $0.1 \mathrm{mHz}$ (days 127-133, with 26 windows of length 40000 s). A later, shorter run, of roughly 2 day duration is included in the time series of Fig. 3 to illustrate the final noise reached at the time of writing, which is significantly lower in the white noisedominated band above $1 \mathrm{mHz}$. We also note that the noise run shown in Fig. 1 used the lowest actuation authority employed thus far and, coincidentally, corresponds with the largest centrifugal force noise correction of the entire data set. We report in Fig. 1 the ASD of the uncorrected data, which shows that, if unaccounted for, the centrifugal force noise would dominate the ASD below $0.5 \mathrm{mHz}$.

Interferometer readout noise in $\Delta x$, with displacement spectral density $S_{\mathrm{IFO}}$, contributes to the ASD of $\Delta g$, according to Eq. (1), a term $S_{\mathrm{IFO}}^{1 / 2}(2 \pi f)^{2}$. Frequencyindependent interferometer noise, limited by noise in the phasemeter [10,11], results in a displacement noise ASD of $(34.8 \pm 0.3) \mathrm{fm} / \sqrt{\mathrm{Hz}}$ and dominates $\Delta g$ above $60 \mathrm{mHz}$.

The raw noise between 20 and $200 \mathrm{mHz}$ exceeds this white noise model in a pronounced "bulge" caused by pickup of the noisy spacecraft motion in the $\Delta x$ interferometric readout. Independent observations of the spacecraft motion show a similar bulge in this band with a maximum jitter of $8-30 \mathrm{~nm} / \sqrt{\mathrm{Hz}}$ on the different axes. By adjusting
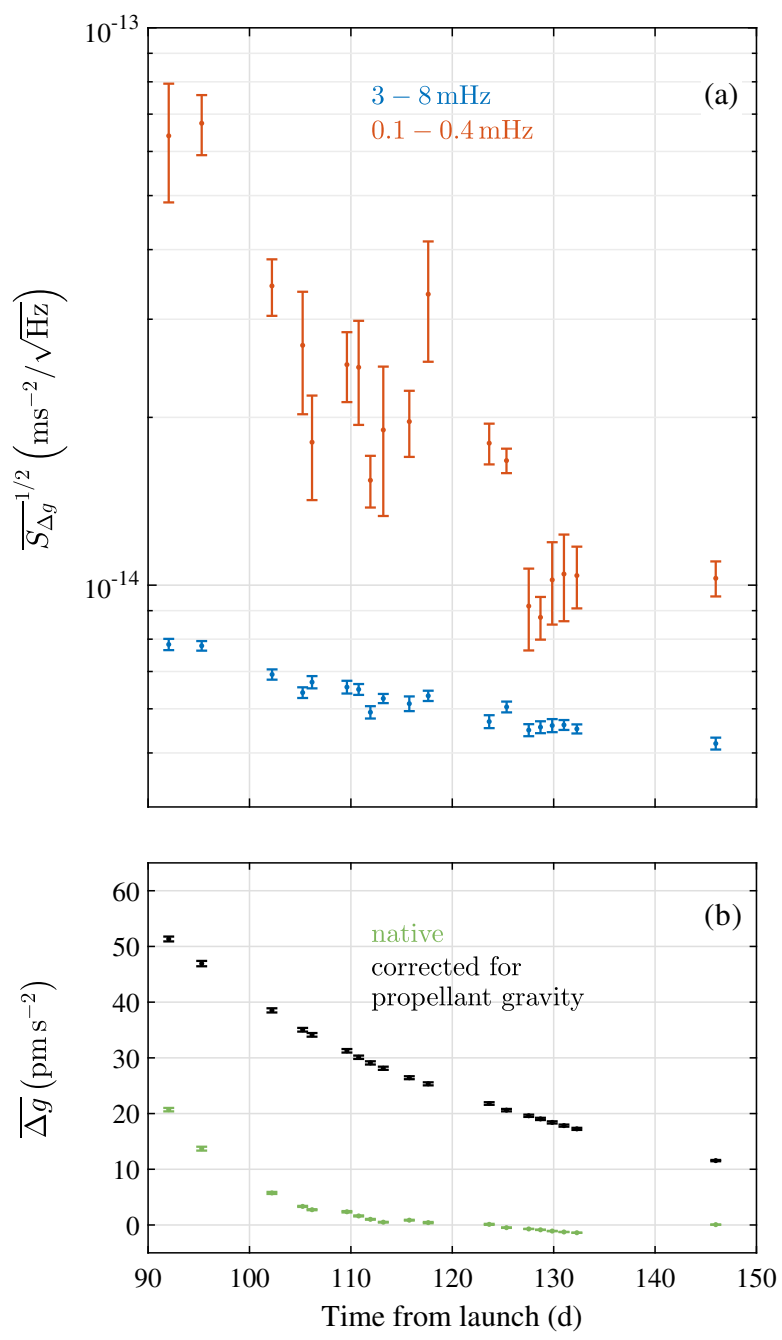

FIG. 3. (a) Square root of the averaged power spectral density of $\Delta g$ in the $3-8 \mathrm{mHz}$ and $0.1-0.4 \mathrm{mHz}$ frequency bands as a function of time. This is calculated from a numerical integration over the relevant band, $\bar{S} \equiv \frac{1}{f_{2}-f_{1}} \int_{f_{1}}^{f_{2}} S(f) d f$. (b) Quasistatic value of $\overline{\Delta g}$ as a function of time, both for the raw time series and, to highlight the unexplained portion of this, the same data corrected for the differential gravitation calculated from the depletion of propellant mass. This correction is large and negative, resulting in consistently higher $\overline{\Delta g}$ values for the corrected data series compared to the native one. All time series are based on $100000 \mathrm{~s}$ groups of $50 \%$ overlapped windows from the 11 noise runs, with $10000 \mathrm{~s}$ windows used for the $3-8 \mathrm{mHz}$ band and $40000 \mathrm{~s}$ for the other three data series. In all cases the errors are assigned based on the scatter between the averaged windows. Gaps in the data series shown here are due to the presence of both station-keeping maneuvers and other experiments.

the angular control setpoints for the TM rotation around the $y$ and $z$ axes for a better beam alignment, this bulge of noise in $\Delta x$ was reduced by roughly a factor of 2 in ASD around $80 \mathrm{mHz}$. The remainder of the pickup that can be seen in Fig. 1 is removed in software by subtracting a linear combination of the measured spacecraft translational and 
rotational acceleration from $\Delta g$. Capacitive sensing provides estimates of the spacecraft translation along the $y$ and $z$ axes and interferometric readouts give measurements of the $x$ translation and rotation around $y$ [7].

The ASD of this corrected time series is shown in light blue in Fig. 1. All fitting coefficients are in the range allowed by a geometrical model of the optical system and by the optical bench design and manufacturing tolerances, and remain stable among runs with the same TM alignment and electrostatic suspension settings. As spacecraft motion is correlated among the different degrees of freedom, the correction procedure is not entirely unambiguous and different combinations of signals also fit the data with acceptable quality.

The bump in the raw data spectrum is visible only because the interferometer noise is well below the required levels, and its correction here is performed only to consolidate our noise model.

We note that the performance of this interferometer is significantly better than required for the local test mass interferometer readout in LISA.

\section{A. Known force noise contributions}

Brownian noise associated with viscous gas damping likely dominates the region of the spectrum where we observe a nearly frequency-independent acceleration noise contribution of $S_{0}^{1 / 2}=(5.57 \pm 0.04) \mathrm{fm} \mathrm{s}^{-2} / \sqrt{\mathrm{Hz}}$ at all frequencies above $1 \mathrm{mHz}$ in the main noise run (Fig. 1), decreasing to $(5.2 \pm 0.1) \mathrm{fm} \mathrm{s}^{-2} / \sqrt{\mathrm{Hz}}$ in the most recent run at day 146. This Brownian noise, with power proportional to $p / \sqrt{m_{0}}$ for a residual gas of molecular weight $m_{0}$ at pressure $p$, has been found [19], both experimentally and in simulations, to be increased by the close proximity of electrode housing surfaces, at only 2.9-4 mm gaps from the $46 \mathrm{~mm}$ cubic TM. With this model, the observed noise floor here could be explained by $9.5 \mu \mathrm{Pa}$ of water, or roughly three times larger pressure for $\mathrm{H}_{2}$.

The mean ASD in this frequency range, and thus also the upper limits on the residual pressure, are observed to decrease over time by roughly a factor of 1.5 over the 55 day period presented. Figure 3 shows the average noise in the 3-8 $\mathrm{mHz}$ band for all noise runs starting from 91 days after launch. Data from the first noise run are excluded here to limit the analysis to points where actuation noise (discussed in the next section) contributes less than 5\% noise power. The data for the $3-8 \mathrm{mHz}$ average are consistent with a $1 /\left(t-t_{0}\right)$ decay, as might be expected for a vacuum system which has only been vented to space since the beginning of February, two weeks before test mass release.

Using the radiometric effect, additional pressure upper limits have been measured with several dedicated thermal gradient experiments. In these tests an oscillating temperature difference, $\Delta T$, is applied across the TM housing with dedicated resistive heaters, and the resulting force coupling, $d F / d \Delta T$ is measured [20]. Measurements are performed over a range of average temperatures (albeit only spread over a few kelvins), to help discriminate the radiometric effect-with $(d F / d \Delta T) \propto(p / T)$ - from more strongly temperature dependent contributions arising from radiation pressure and temperature dependent outgassing [20]. These measurements of $d F / d \Delta T$ also show a significant decay over time and place an absolute average pressure upper limit of roughly $22 \mu \mathrm{Pa}$ at the time of the main noise measurement shown in Fig. 1. Though consistent with a Brownian noise floor due to a decaying atmosphere of water, further measurement and analysis are under way to place a more stringent estimate of the residual pressure and other possible noise sources in this $\mathrm{mHz}$ frequency band.

An increase in the noise is clearly visible in Fig. 1 at frequencies below $0.5 \mathrm{mHz}$. This source of noise increases above the white noise background with decreasing frequency, slightly exceeding $10 \mathrm{fm} \mathrm{s}^{-2} / \sqrt{\mathrm{Hz}}$ from 0.1 to $0.3 \mathrm{mHz}$. The remainder of the mission's experimental campaign will be largely dedicated to establishing a quantitative experimental model for this low-frequency noise, along the lines of the analysis in Ref. [9] and supplemented by ground measurements where available.

A partial analysis is possible for many sources with the data already measured. For instance, actuation noise from fluctuations in the electrostatic force applied on TM2 was expected to be the dominant noise around $1 \mathrm{mHz}$ [9], increasing with both the needed applied dc forces and the force and torque authorities, as discussed in Sec. II A.

The quasi-dc differential acceleration has varied over the mission, decreasing from a maximum of slightly more than $20 \mathrm{pm} / \mathrm{s}^{2}$ at the start of operations (see the "native" $\overline{\Delta g}$ data in Fig. 3(b)). This is well below the $600 \mathrm{pm} / \mathrm{s}^{2}$ budgeted for the applied force level due to the successful gravitational balancing of the spacecraft [16], and has allowed us to reduce the force authority along $x$ to roughly $25 \mathrm{pm} / \mathrm{s}^{2}$ during the measurement shown in Fig. 1. At this level, actuation noise is a negligible contributor around $1 \mathrm{mHz}$, so we chose not to reduce the control authority any further to maintain an ample margin. The actuation noise measured around $1 \mathrm{mHz}$ with much larger authorities $\left(1.1 \mathrm{~nm} / \mathrm{s}^{2}\right)$, prior to the measurements shown in Figs. 1 and 3, was consistent with the roughly $5 \mathrm{ppm} / \sqrt{\mathrm{Hz}}$ actuation stability measured on ground [9]. Reliable projection to the $0.1 \mathrm{mHz}$ noise in the current low authority configuration is not possible from the high authority measurements, due to the low-frequency noise nonstationarity — a steady improvement to be discussed shortly-early in the mission, and low-frequency actuation noise will be the target of an upcoming measurement campaign.

At low frequencies, various other noise sources that contribute to the LPF error budget (and to any future LISAlike mission) need to be considered. A partial list of sources 
and the current status of our experimental knowledge is summarized here:

TM charge fluctuations $(\delta q)$ from cosmic rays, couple to stray electrostatic fields to produce force noise, with $\delta F=(\partial F / \partial q) \delta q$. Measured values of $\partial F / \partial q$-essentially the average stray field inside the electrode housing [21] combined with measured long-term fluctuations of the TM charge, yield an estimate of roughly $3 \mathrm{fm} \mathrm{s}^{-2} / \sqrt{\mathrm{Hz}}$ at $0.1 \mathrm{mHz}$ for this effect. Full compensation of $\partial F / \partial q$ with applied voltages will be done now with LPF and, if needed, in LISA. Additionally, an upcoming measurement of acceleration noise with an intentionally charged TM will quantify the role of temporal fluctuations in the stray electrostatic fields themselves.

Thermal gradient effects can produce time-varying stray forces, as mentioned in the discussion of Brownian noise. Measured values of $d F / d \Delta T \simeq 20 \mathrm{pms}^{-2} / \mathrm{K}$ and estimates of the temperature fluctuations from thermistors [22] (measured to be $10 \mu \mathrm{K} / \sqrt{\mathrm{Hz}}$ at $1 \mathrm{mHz}$, and $50 \mu \mathrm{K} / \sqrt{\mathrm{Hz}}$ at $0.1 \mathrm{mHz}$ ) put an upper limit on this effect of $1 \mathrm{fm} \mathrm{s}^{-2} / \sqrt{\mathrm{Hz}}$ at $0.1 \mathrm{mHz}$.

Laser radiation pressure fluctuations cause a fluctuating force on both TMs. Measured laser intensity fluctuations of the order of $0.4 \mu \mathrm{W} / \sqrt{\mathrm{Hz}}$ indicate a force noise contribution of roughly $2 \mathrm{fm} \mathrm{s}^{-2} / \sqrt{\mathrm{Hz}}$.

Magnetic force effects are analyzed with the magnetic field fluctuations measured onboard by four different magnetometers [5], and their ASD is $\simeq 100 \mathrm{nT} / \sqrt{\mathrm{Hz}}$ at $0.1 \mathrm{mHz}$. This, together with the fact that fluctuating field is uniform in space, indicates that the fluctuations arise from the interplanetary field. In a uniform field the magnetic force is dominated by the interaction of the magnetic moment induced in the TM by the fluctuating field, with the static magnetic gradient at the TM location. Pending a planned onboard calibration, we can only use upper limits to the gradients derived from ground measurements [9] for the calculation of the ASD of the force. From these we get an estimate consistent with the full excess noise observed at $0.1 \mathrm{mHz}$. However, the lack of significant linear correlation between the measured magnetic field time series and $\Delta g$ places a more stringent upper limit to this contribution at $3 \mathrm{fm} \mathrm{s}^{-2} / \sqrt{\mathrm{Hz}}$.

\section{B. Additional observations}

In addition to the steady state levels of these and other noise sources, we add that the low-frequency noise has been observed to decrease by about 1 order of magnitude in the $0.1-0.4 \mathrm{mHz}$ band (see Fig. 3(a)) over the first 55 days of operations leading up to the measurement presented in Fig. 1.

We do not have an explanation for the large value of the low-frequency noise at the beginning of operations, nor for its improvement over time. It may, however, be related to a drift in the "quasistatic" value of $\Delta g$ (see Fig. 3(b)).
The rate of change of $\Delta g$, not fully explained by the selfgravity change from propellant depletion, is also observed to decrease by a factor of 4 in the first two months of operations. There may be a relaxation mechanism, perhaps related to the pressure decay or mechanical stress release, responsible for both the decaying drift in $\Delta g$ and the improving noise spectrum at frequencies near $0.1 \mathrm{mHz}$. This effect, and the future evolution of the system, will be studied during the rest of the mission.

In conclusion, compared to the LISA single TM acceleration noise requirements of $3 \mathrm{fm} \mathrm{s}^{-2} / \sqrt{\mathrm{Hz}}$, the current LPF results are a factor of 1.25 higher at $\mathrm{mHz}$ frequencies and about a factor of 3 at $0.1 \mathrm{mHz}$. At all frequencies the noise has been improving up to the point of writing.

A fraction of the very low-frequency noise can be explained by the terms in our noise budget that have been measured to date. Further measurements during the rest of the mission aim to consolidate the noise model and establish what, if any, mitigation strategy will be needed for LISA.

\section{IMPACT OF THE LISA PATHFINDER RESULT}

LISA Pathfinder has demonstrated in its first 55 days of science operations the functionality of a system quite similar to that needed aboard each satellite of a space-based GW observatory: 15 degree-of-freedom control including two free-falling test masses, a drag-free satellite with cold gas thrusters, electrostatic force actuation, contactless test-mass charge control, and short arm interferometric local displacement and attitude readout, the first of its kind in orbit. All of these subsystems have performed as expected and could be used for LISA. The system has proven to be extremely stable, reliable, and robust, never falling out of the science measurement mode and with continuous, $100 \%$ duty cycle data stretches limited to two weeks only due to maneuvers needed to maintain the L1 orbit.

The performance obtained and the corresponding physical model for the LPF differential acceleration measurement between free-falling test masses, are already at a level that allows implementation of a successful GW observatory in space; both have far-reaching consequences for gravitational experimentation in general. The current LPF noise floor in the $\mathrm{mHz}$ band is $4-5$ orders of magnitude below the best levels achieved to date by the GOCE geodesy mission [23]. The results from LPF show that sub-femto- $g$ differential accelerometry is possible far away from the low earth-orbit gravity gradient. The corresponding required reduction of acceleration noise arising from spurious forces is achieved by design, the main features of which are kilogram sized TMs, millimeter gaps, the absence of applied dc voltages and discharge wires, and the measurement of relative motion with a high precision interferometer. 
The consequences are even more striking for the observation of GWs from space. If transferred to the LISA observatory configuration, the acceleration noise performance already achieved on LISA Pathfinder would allow for an observatory performance very near the original LISA mission goals [2]. Heavier black hole mergers dominated by low-frequency emission, such as $10^{7} \mathrm{M}_{\odot}$ total mass at redshift $z=3$, will be visible with a signal-to-noise ratio (SNR) of 1400, compared with 2000 for the LISA reference performance; lighter systems $\left(10^{6} \mathrm{M}_{\odot}\right)$ at the same distance will give an SNR of 5000, which is within several percent of the LISA performance. Science from extreme mass-ratio inspirals and coalescing compact binaries in our galaxy will give essentially the same SNR as in LISA. The LISA Pathfinder performance is therefore fully in line with the mission concept put forward in "The Gravitational Universe" [24], which was selected by ESA for the third large mission (L3) of the Cosmic Vision programme. LISA Pathfinder represents a major step forward in demonstrating the proposed measurement concept.

\section{ACKNOWLEDGMENTS}

This work has been made possible by the LISA Pathfinder mission, which is part of the space-science program of the European Space Agency.

The French contribution has been supported by CNES (Accord Specific de projet CNES 1316634/CNRS 103747), the CNRS, the Observatoire de Paris and the University Paris-Diderot. E. P. and H. I. would also like to acknowledge the financial support of the UnivEarthS Labex program at Sorbonne Paris Cité (ANR-10-LABX-0023 and ANR-11IDEX-0005-02).

The Albert-Einstein-Institut acknowledges the support of the German Space Agency, DLR. The work is supported by the Federal Ministry for Economic Affairs and Energy based on a resolution of the German Bundestag (FKZ 50OQ0501 and FKZ 50OQ1601).

The Italian contribution has been supported by Agenzia Spaziale Italiana and Instituto Nazionale di Fisica Nucleare.

The Spanish contribution has been supported by Contracts No. AYA2010-15709 (MICINN), No. ESP2013-47637-P, and No. ESP2015-67234-P (MINECO). M. N. acknowledges support from Fundacion General CSIC (Programa ComFuturo). F. R. acknowledges support from a Formación de Personal Investigador (MINECO) contract.

The Swiss contribution acknowledges the support of the Swiss Space Office (SSO) via the PRODEX Programme of ESA. L. F. acknowledges the support of the Swiss National Science Foundation.

The UK groups wish to acknowledge support from the United Kingdom Space Agency (UKSA), the University of Glasgow, the University of Birmingham, Imperial College, and the Scottish Universities Physics Alliance (SUPA).

J. I. T. and J.S. acknowledge the support of the U.S. National Aeronautics and Space Administration (NASA).
*Deceased September 2012.

Current address: University of Florida, Gainesville, FL 32611, USA.

Current address: Department of Physics, University of Oxford, Keble Road, Oxford, OX1 3RH United Kingdom.

${ }^{\S}$ Current address: OHB System AG, Universitätsallee 27-29, D-28359 Bremen, Germany.

"Current address: National Institute for Standards and Technology and JQI, University of Maryland, 100 Bureau Drive, MS 8212 Gaithersburg, MD 20899, USA.

"Current address: Thales Alenia Space, 5 All des Gabians, BP 99-06156 Cannes, France.

** Current address: National Institute for Standards and Technology, 325 Broadway, Boulder, CO 80305, USA.

${ }^{\dagger}$ Current address: Deutsches Zentrum für Luft- und Raumfahrt, Robert-Hooke-Str. 7, 28359 Bremen, Germany. \#Current address: Institute of Geodesy and Geophysics, CAS, No. 340 Xudong Street, Wuhan 430077, China.

${ }^{\S}$ Current address: Jena-Optronik GmbH, Otto-EppensteinStrasse 3, 07745 Jena, Germany.

[1] P. McNamara, S. Vitale, and K. Danzmann, Classical Quantum Gravity 25, 114034 (2008).

[2] K. Danzmann et al., LISA: Unveiling a Hidden Universe Report No. ESA/SRE2011(2011)3, 2011.

[3] G. Congedo, R. Dolesi, M. Hueller, S. Vitale, and W. J. Weber, Phys. Rev. D 88, 082003 (2013).

[4] K. Danzmann and the LISA Study Team, Laser Interferometer Space Antenna: A Cornerstone Mission for the Observation of Gravitational Waves, ESA System and Technology Study Report No. ESA-SCI (2000).

[5] S. Anza et al., Classical Quantum Gravity 22, S125 (2005).

[6] W. Fichter, A. Schleicher, N. Brandt, S. Vitale, and D. Bortoluzzi, in Guidance, Navigation and Control Systems, ESA Special Publication (ESA, Noordwijk, the Netherlands, 2006), Vol. 606, p. 56.1.

[7] R. Dolesi et al., Classical Quantum Gravity 20, S99 (2003).

[8] T. J. Sumner, D. N. A. Shaul, M. O. Schulte, S. Waschke, D. Hollington, and H. Araújo, Classical Quantum Gravity 26, 094006 (2009).

[9] F. Antonucci et al., Classical Quantum Gravity 28, 094002 (2011).

[10] G. Heinzel, C. Braxmaier, R. Schilling, A. Rüdiger, D. Robertson, M. te Plate, V. Wand, K. Arai, U. Johann, and K. Danzmann, Classical Quantum Gravity 20, S153 (2003).

[11] G. Heinzel, V. Wand, A. García, O. Jennrich, C. Braxmaier, D. Robertson, K. Middleton, D. Hoyland, A. Rüdiger, R. Schilling, U. Johann, and K. Danzmann, Classical Quantum Gravity 21, S581 (2004).

[12] H. Audley et al., Classical Quantum Gravity 28, 094003 (2011).

[13] W. Fichter, P. Gath, S. Vitale, and D. Bortoluzzi, Classical Quantum Gravity 22, S139 (2005).

[14] P. G. Maghami, T. T. Hyde, and J. Kim, Classical Quantum Gravity 22, S421 (2005).

[15] L. Carbone, A. Cavalleri, R. Dolesi, C. D. Hoyle, M. Hueller, S. Vitale, and W. J. Weber, Phys. Rev. Lett. 91, 151101 (2003).

[16] V. Ferroni, arXiv:1604.08360.

[17] S. Vitale et al., Phys. Rev. D 90, 042003 (2014). 
[18] P. D. Welch, IEEE Trans. Audio Electroacoust. 15, 70 (1967).

[19] A. Cavalleri, G. Ciani, R. Dolesi, A. Heptonstall, M. Hueller, D. Nicolodi, S. Rowan, D. Tombolato, S. Vitale, P. J. Wass, and W. J. Weber, Phys. Rev. Lett. 103, 140601 (2009).

[20] L. Carbone, A. Cavalleri, G. Ciani, R. Dolesi, M. Hueller, D. Tombolato, S. Vitale, and W. J. Weber, Phys. Rev. D 76, 102003 (2007).
[21] F. Antonucci, A. Cavalleri, R. Dolesi, M. Hueller, D. Nicolodi, H. B. Tu, S. Vitale, and W. J. Weber, Phys. Rev. Lett. 108, 181101 (2012).

[22] J. Sanjuán, A. Lobo, M. Nofrarias, J. Ramos-Castro, and P. J. Riu, Rev. Sci. Instrum. 78, 104904 (2007).

[23] R. Rummel, W. Yi, and C. Stummer, J. Geodes. 85, 777 (2011).

[24] P. Amaro Seoane et al., arXiv:1305.5720. 